Engineering Optimization

Vol. 00, No. 00, Month 200x, 1-11

\title{
On the Use of Mathematical Programs with Complementary Constraints in Combined Topological and Parametric Design of Biochemical Enzyme Networks (Supplemental Materials)
}

\author{
Tinghao Guo and James T. Allison* \\ Industrial and Enterprise Systems Engineering \\ Institute for Genomic Biology \\ University of Illinois at Urbana-Champaign \\ Urbana IL, 61801 \\ (Received April 20XX)
}

discretized state variable trajectories

defect constraint

concentration of node $\mathrm{A}$

concentration of node $\mathrm{B}$

concentration of node $\mathrm{C}$

concentration of node D

error between steady state output before and after the input change

activating basal enzyme, where $i=A, B, C, D$ and $E_{i}=0.5$

deactivating basal enzyme, where $i=A, B, C, D$ and $F_{i}=0.5$

input $I_{1}=0.5$ before $t=40 \mathrm{~s}$

input $I_{2}=0.6$ after $t=40 \mathrm{~s}$

catalytic rate constant, where $i=A, B, C, D$

Michaelis-Menten constant, where $i=A, B, C, D$

the number of divided segments or intervals in the time domain

steady state output before the input change

steady state output after the input change

This supplementary document provides additional details relevant to the the article

'On the Use of Mathematical Programs with Complementary Constraints in Combined

Topological and Parametric Design of Biochemical Enzyme Networks' (Guo and Allison

2016). Models and optimization formulations are described in full detail, and the nested

*Corresponding author. Email: jtalliso@illinois.edu

ISSN: 0305-215X print/ISSN 1029-0273 online 
solution strategy (a baseline method for comparison to the new DT-MPCC method) is presented. In addition, numerical study results are presented in detail, including 3 - and 4-node problem results, equilibrium point studies, and sensitivity analyses. These results can be replicated using the code provided at the URL indicated in (Allison 2016).

\section{MPCC Formulation: Three-node Problem}

The design problem was presented in simplified form in Eq. (10) of Guo and Allison (2016). Here we present the fully-detailed formulation of this design problem with complementarity constraints specifically for the dynamic three-node enzyme network:

$$
\begin{aligned}
& \min _{\mathbf{A}, \mathbf{B}, \mathbf{C}, \mathbf{k}, \mathbf{K}} \quad E=\left|\frac{\left(O_{2}-O_{1}\right) / O_{1}}{\left(I_{2}-I_{1}\right) / I_{1}}\right| \\
& \text { s.t. }\left|\frac{\left(O_{\text {peak }}-O_{1}\right) / O_{1}}{\left(I_{2}-I_{1}\right) / I_{1}}\right| \geq 1 \\
& \frac{d A}{d t}=I k_{I A} \frac{(1-A)}{(1-A)+K_{I A}}+B k_{B A} \frac{(1-A)}{(1-A)+K_{B A}}+C k_{C A} \frac{(1-A)}{(1-A)+K_{C A}} \\
&-B k_{B A}^{\prime} \frac{A}{A+K_{B A}^{\prime}}-C k_{C A}^{\prime} \frac{A}{A+K_{C A}^{\prime}}-F_{A} k_{F_{A} A}^{\prime} \frac{A}{A+K_{F_{A} A}^{\prime}} \\
& \frac{d B}{d t}=A k_{A B} \frac{(1-B)}{(1-B)+K_{A B}}+C k_{C B} \frac{(1-B)}{(1-B)+K_{C B}}+E_{B} k_{E_{B} B} \frac{(1-B)}{(1-B)+K_{E_{B} B}} \\
&-A k_{A B}^{\prime} \frac{B}{B+K_{A B}^{\prime}}-C k_{C B}^{\prime} \frac{B}{B+K_{C B}^{\prime}}-F_{B} k_{F_{B} B}^{\prime} \frac{B}{B+K_{F_{B} B}^{\prime}} \\
& \frac{d C}{d t}=A k_{A C} \frac{(1-C)}{(1-C)+K_{A C}}+B k_{B C} \frac{(1-C)}{(1-C)+K_{B C}}+E_{C} k_{E_{C} C} \frac{(1-C)}{(1-C)+K_{E_{C} C}} \\
&-A k_{A C}^{\prime} \frac{C}{C+K_{A C}^{\prime}}-B k_{B C}^{\prime} \frac{C}{C+K_{B C}^{\prime}}-F_{C} k_{F_{C} C}^{\prime} \frac{C}{C+K_{F_{C} C}^{\prime}} \\
& 0 \leq \mathbf{k}_{\mathbf{1}} \perp \mathbf{k}_{\mathbf{2}} \geq 0,0 \leq \mathbf{k} \leq 10,0 \leq \mathbf{K} \leq 10
\end{aligned}
$$

Here the term $I k_{I A} \frac{(1-A)}{(1-A)}$ is an input to node $A$. Equations (1)-(3) here correspond to $f_{A}(A), F_{B}(B)$, and $f_{C}(C)$ in Eq. (9) in Guo and Allison (2016). The vectors $\mathbf{k}_{\mathbf{1}}, \mathbf{k}_{\mathbf{2}}, \mathbf{k}$, and $\mathbf{K}$ are defined for convenience as follows:

$$
\begin{aligned}
\mathbf{k}_{\mathbf{1}}= & \left(k_{B A}, k_{C A}, k_{B A}^{\prime}, k_{C A}^{\prime}, k_{A B}, k_{C B}, k_{A B}, k_{C B}, k_{A B}^{\prime}, k_{C B}^{\prime}, k_{A C}, k_{B C}, k_{A C}, k_{B C}, k_{A C}^{\prime}, k_{B C}^{\prime}\right) \\
\mathbf{k}_{\mathbf{2}}= & \left(k_{B A}^{\prime}, k_{C A}^{\prime}, k_{F_{A} A}^{\prime}, k_{F_{A} A}^{\prime}, k_{A B}^{\prime}, k_{C B}^{\prime}, k_{E_{B} B}, k_{E_{B} B}, k_{F_{B} B}^{\prime}, k_{F_{B} B}^{\prime}, k_{A C}^{\prime}, k_{B C}^{\prime}, k_{E_{C} C}, k_{E_{C} C},\right. \\
& \left.k_{F_{C} C}^{\prime}, k_{F_{C} C}^{\prime}\right) \\
\mathbf{k}= & \left(k_{I A}, k_{B A}, k_{C A}, k_{B A}^{\prime}, k_{C A}^{\prime}, k_{F_{A} A}^{\prime}, k_{A B}, k_{C B}, k_{E_{B} B}, k_{A B}^{\prime}, k_{C B}^{\prime}, k_{F_{B} B}^{\prime}, k_{A C}, k_{B C}, k_{E_{C} C}, k_{A C}^{\prime},\right. \\
& \left.k_{B C}^{\prime}, k_{F_{C} C}^{\prime}\right) \\
\mathbf{K}= & \left(K_{I A}, K_{B A}, K_{C A}, K_{B A}^{\prime}, K_{C A}^{\prime}, K_{F_{A} A}^{\prime}, K_{A B}, K_{C B}, K_{E_{B} B}, K_{A B}^{\prime}, K_{C B}^{\prime}, K_{F_{B} B}^{\prime}, K_{A C}, K_{B C},\right. \\
& \left.K_{E_{C} C}, K_{A C}^{\prime}, K_{B C}^{\prime}, K_{F_{C} C}^{\prime}\right) .
\end{aligned}
$$




\section{Nested Optimization Formulation: Four-node Problem}

For the nested optimization strategy, discrete design variables $x_{i j}$ are defined that represent network topology:

$$
x_{i j}= \begin{cases}+1 & \text { a positive regulation link from node } i \text { to node } j \\ 0 & \text { no regulation between nodes } i \text { and } j \\ -1 & \text { a negative regulation link from node } i \text { to node } j\end{cases}
$$

where $\{i, j\} \in\{A, B, C, D\} \times\{A, B, C, D\}$, for $i \neq j$. The outer-loop problem is formulated as integer program:

$$
\begin{array}{ll}
\min _{\mathbf{x}} & \hat{E}_{*}(\mathbf{x}) \\
\text { s.t. } & -3 \leq x_{B A}+x_{C A}+x_{D A} \leq 3 \\
-3 & \leq x_{A B}+x_{C B}+x_{D B} \leq 3 \\
- & \leq x_{A C}+x_{B C}+x_{D C} \leq 3 \\
- & \leq x_{A D}+x_{B D}+x_{C D} \leq 3 \\
& x_{i j} \in\{-1,0,1\}, \text { for } i, j \in\{A, B, C, D\} .
\end{array}
$$

$\hat{E}_{*}$ is the minimum squared error identified by the inner loop for a given topology $\mathbf{x}$. More precisely, $\hat{E}_{*}$ is the optimal objective function value that results from solving the inner loop problem:

$$
\begin{array}{cl}
\min _{\mathbf{k}, \mathbf{K}, \boldsymbol{\Xi}} \quad \hat{E}=\left(\frac{\left(O_{2}-O_{1}\right) / O_{1}}{\left(I_{2}-I_{1}\right) / I_{1}}\right)^{2} \\
\text { s.t. } & \left|\frac{\left(O_{\text {peak }}-O_{1}\right) / O_{1}}{\left(I_{2}-I_{1}\right) / I_{1}}\right| \geq 1 \\
& \boldsymbol{\zeta}_{i}(\boldsymbol{\Xi}, \mathbf{k}, \mathbf{K})=\mathbf{0} \\
& 0 \leq \mathbf{k} \leq 10, \quad 0 \leq \mathbf{K} \leq 100 \\
\text { where } & i=1,2, \ldots, n_{t}-1, n_{t} .
\end{array}
$$

In the outer-loop problem, the topological design vector $\mathbf{x}$ specifies the existence of regulations between nodes. If the candidate solution $\mathbf{x}$ produces a topology where a node has only incoming positive or negative regulations, it is assumed that a basal enzyme level will deactivate $\left(F_{i}=0.5\right)$ or activate $\left(E_{i}=0.5\right)$ that node. For a given network topology, the inner loop problem is solved using the direct transcription approach. Complementarity constraints (CCs) are not needed in the inner loop problem since topology is fixed and specified by the outer loop problem. Because the same objective function is used for inner and outer loops, the nested optimization formulation is mathematically equivalent to the simultaneous DT-MPCC formulation. The primary difference between the inner- and outer-loop problems is the set of optimization variables used. The innerloop problem manages all continuous design and state variables, whereas the outer-loop problem manages discrete topological design variables. This decomposition allows use of 
appropriate algorithms for each problem.

\section{Numerical Results for the Three-node Enzyme Networks}

The optimal design parameters for the three-node enzyme network obtained using the single shooting method and simultaneous DT approach are reported below. Figures 1 and 2 illustrate the topologies and the corresponding system responses for these solutions.



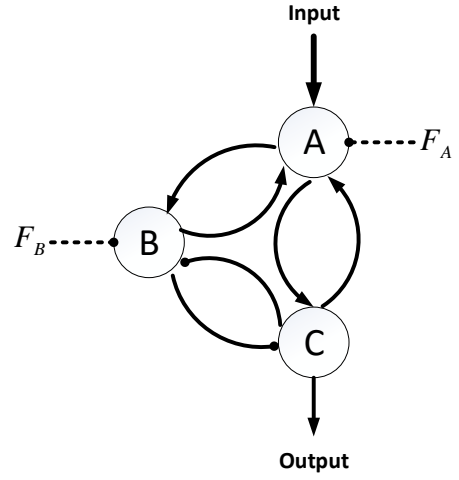

(a)

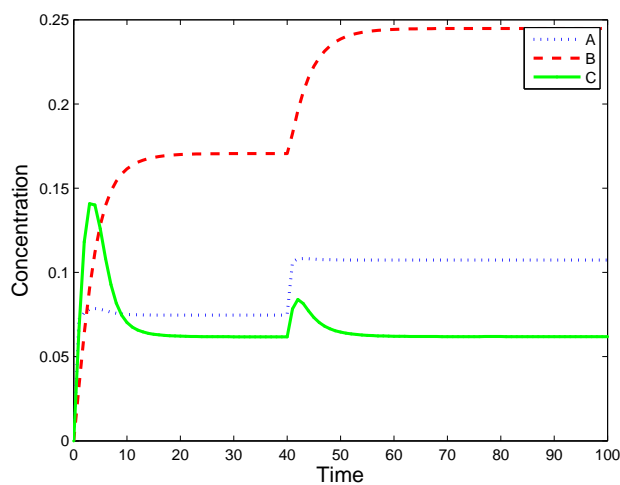

(b)

Figure 1. Resulting three-node topology and system response obtained using the single shooting method

\section{Numerical Results for the Four-node Enzyme Networks}

The optimal design parameters of the four-node enzyme networks obtained using the nested optimization method and simultaneous DT approach are reported below. Figures 3 and 4 illustrate the topologies and their corresponding system responses for these solutions. 


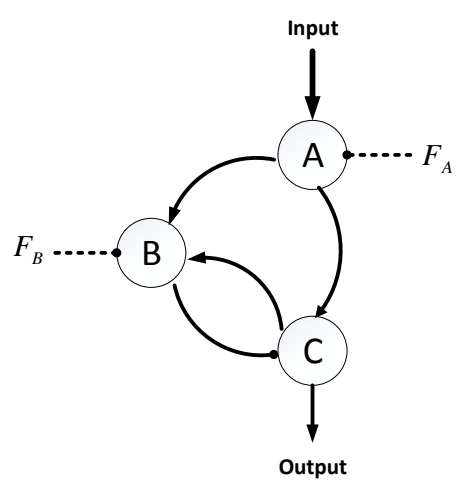

(a)

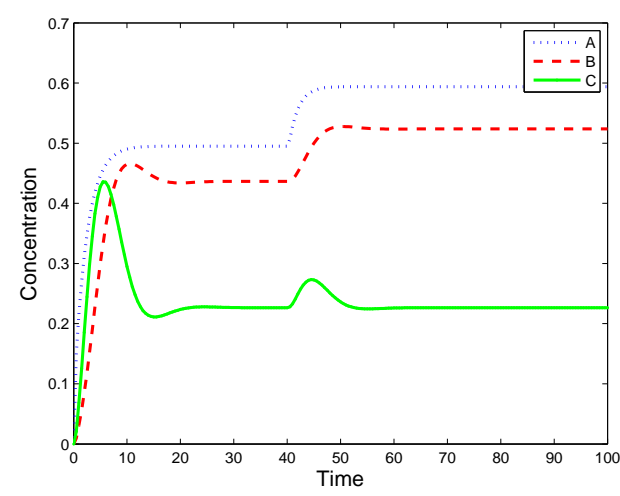

(b)

Figure 2. Resulting three-node topology and system response obtained using the simultaneous DT approach

$$
\begin{aligned}
\mathbf{x}_{\text {nested }^{*}=} & \left(x_{B A}, x_{C A}, x_{D A}, x_{A B}, x_{C B}, x_{D B}, x_{A C}, x_{B C}, x_{D C}, x_{A D}, x_{B D}, x_{C D}\right) \\
= & (-1,0,0,1,1,1,-1,-1,-1,1,-1,0) \\
\mathbf{k}_{\text {nested }^{*}=} & \left(k_{I A}, k_{B A}^{\prime}, k_{A B}, k_{C B}, k_{D B}, k_{F B B}^{\prime}, k_{A C}^{\prime}, k_{B C}^{\prime}, k_{D C}^{\prime}, k_{E C C}, k_{A D}, k_{B D}^{\prime}\right) \\
= & (1.51,1.66,1.23,0.25,0.07,0.67,3.03,4.45,3.69,6.76,1.39,1.36) \\
\mathbf{K}_{\text {nested }^{*}=} & \left(K_{I A}, K_{B A}^{\prime}, K_{A B}, K_{C B}, K_{D B}, K_{F B B}^{\prime}, K_{A C}^{\prime}, K_{B C}^{\prime}, K_{D C}^{\prime}, K_{E C C}, K_{A D}, K_{B D}^{\prime}\right) \\
= & (0.08,0.07,0.84,5.98,5.30,0.20,5.27,4.61,5.02,3.00,0.06,0.04) \\
\mathbf{k}_{\mathbf{D T}^{*}}= & \left(k_{I A}, k_{B A}, k_{C A}, k_{D A}, k_{B A}^{\prime}, k_{C A}^{\prime}, k_{D A}^{\prime}, k_{F_{A} A}^{\prime},\right. \\
& k_{A B}, k_{C B}, k_{D B}, k_{E_{B} B}, k_{A B}^{\prime}, k_{C B}^{\prime}, k_{D B}^{\prime}, k_{F_{B} B}^{\prime}, \\
& k_{A C}, k_{B C}, k_{D C}, k_{E_{C} C}, k_{A C}^{\prime}, k_{B C}^{\prime}, k_{D C}^{\prime}, k_{F_{C} C}^{\prime}, \\
& \left.k_{A D}, k_{B D}, k_{C D}, k_{E_{D} D}, k_{A D}^{\prime}, k_{B D}^{\prime}, k_{C D}^{\prime} k_{F_{D} D}^{\prime}\right) \\
= & (1.16,0,0,0,0.36,0.33,1.26,0,0.28,0,0.22,0,0,0.26,0,0, \\
& 0.08,0.04,0.24,0,0,0,0,0.24,0.46,0.44,0,0,0,0,0.57,0) \\
\mathbf{K}_{\mathbf{D} \mathbf{T}^{*}}= & \left(K_{I A}, K_{B A}, K_{C A}, K_{D A}, K_{B A}^{\prime}, K_{C A}^{\prime}, K_{D A}^{\prime}, K_{F_{A} A}^{\prime},\right. \\
& K_{A B}, K_{C B}, K_{D B}, K_{E_{B} B}, K_{A B}^{\prime}, K_{C B}^{\prime}, K_{D B}^{\prime}, K_{F_{B} B}^{\prime}, \\
& K_{A C}, K_{B C}, K_{D C}, K_{E_{C} C}, K_{A C}^{\prime}, K_{B C}^{\prime}, K_{D C}^{\prime}, K_{F_{C} C}^{\prime}, \\
& \left.K_{A D}, K_{B D}, K_{C D}, K_{E_{D} D}, K_{A D}^{\prime}, K_{B D}^{\prime}, K_{C D}^{\prime} K_{F_{D} D}^{\prime}\right) \\
= & (0.24,2.38,1.79,2.51,0.19,1.40,0.2,1.28,0.18 .1 .59,0.73,0.6,1.05,0.02,1.15,0.63 \\
& 0.15,1.43,0.06,1.19,0.86,0.13,0.29,0.16,0.09,1.77,1.75,1.20,0.85,0.32,0,0.02) .
\end{aligned}
$$




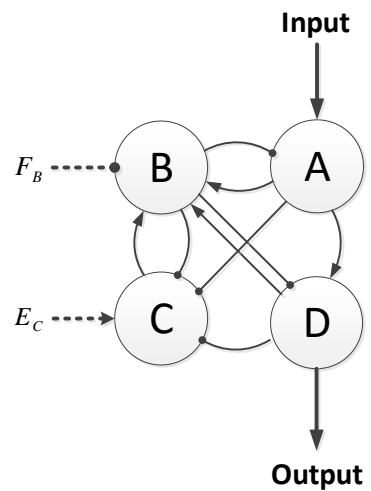

(a)



(b)

Figure 3. Resulting four-node topology and system response obtained using nested optimization

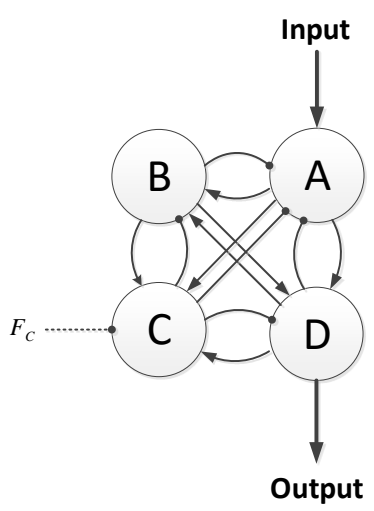

(a)

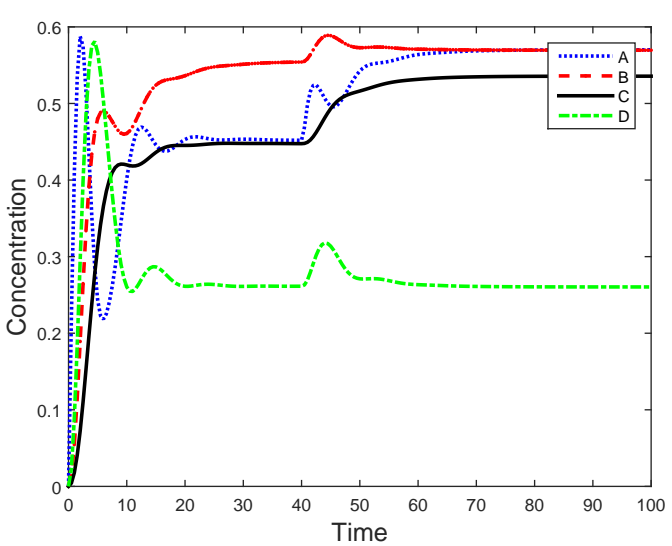

(b)

Figure 4. Resulting four-node topology and system response obtained using the simultaneous DT approach

\section{Steady State Concentrations}

Table 1 reports the equilibrium solutions found by solving the following equation for the optimal three- and four-node designs:

$$
\frac{d T}{d t}=0, \quad \text { where } T=A, B, C \text { or } D
$$

Note that node concentrations range between 0 and 1 due to assumptions used within the mathematical model. The input value used here is $I=0.6$. Multiple equilibrium solutions can exist for nonlinear systems; these multiple solutions are reported in the table below. For both the three- and four-node cases, however, only one of these equilibria is valid for each case. The others are physically infeasible since one of the concentration values lies outside the range of $[0,1]$. The valid steady states (marked with $*$ ) are $[0.5948,0.5251,0.2287]$ and $[0.5857,0.5560,0.5491,0.2538]$ for three node and four-node network design in Fig. 2(a) and Fig. 4(a), respectively. 
Table 1. Multiple steady-state concentrations identified for 3- and 4-node networks. Negative concentration values are not physically meaningful.

\begin{tabular}{l}
\hline \hline Fig. 2(a) (3-node): $\quad[A, B, C]=\left\{\begin{array}{l}{[0.5948,0.5251,0.2287]^{*}} \\
{[0.5948,0.2568,-0.1140]} \\
{[0.5948,-0.2138,1.050]}\end{array}\right.$ \\
Fig. 4(a) (4-node): $[A, B, C, D]=\left\{\begin{array}{l}{[0.5857,0.5560,0.5491,0.2538]^{*}} \\
{[1.2279,-0.0162,1.1530,-12.2354]} \\
{[1.2320,-0.0175,1.1520,-18.6798]} \\
{[1.2260,-0.0160,1.0023,-10.4819]} \\
{[1.0670,-0.2532,1.0362,-0.3074]} \\
{[1.0974,-0.2278,1.2042,-0.5257]}\end{array}\right.$ \\
\hline
\end{tabular}

\section{Sensitivity Analyses}

Sensitivity analyses are presented in this section. The partial derivatives of the output with respect to model parameters are quantified. More specifically, the first-order local sensitivity coefficients for the three-node and four-node network designs can be written as:

$$
\frac{\partial T}{\partial k_{i, j}}, \quad \frac{\partial T}{\partial K_{i, j}}, \text { for } T=C \text { or } D, \text { and } i, j=A, B, C
$$

Figure 5 illustrates how the output trajectory changes with respect to unit changes in a subset of parameters. Two sets of parameters - catalytic rate constants $k_{A B}, k_{F B B}^{\prime}, k_{A C}, k_{B C}$ and Michaelis-Menton constants $K_{A B}, K_{F_{B} B}^{\prime}, K_{A C}, K_{B C}$ - exhibit relatively high sensitivity in the three-node network of Fig. 2(a). These parameters pertain to a path $A \rightarrow B-\bullet C$ and $A \rightarrow C$, an incoherent feedforward loop that was discovered as a core regulatory motif by Ma et al. (2009). Similarly, for the four-node system illustrated in Fig. 4(a), the catalytic rate constants $k_{A C}, k_{D C}, k_{F_{C} C}^{\prime}, k_{A D}, k_{C D}$, and Michaelis-Menton constants $K_{C D}^{\prime}$, play important roles in maintaining the stability of output node $D$. These parameters are associated with the paths $A \rightarrow C \rightarrow D$ and $A \rightarrow D$, which together form an incoherent feedforward loop within the four-node enzyme network. This implies that the incoherent feedforward loop is an important topology for maintaining system stability.

Figures 7(a) and 7(b) illustrate the relationship between parameters and the error $E$ for the optimal topologies shown in Figs. 2(a) and 4(a). Here a $\pm 20 \%$ perturbation about the nominal values of $k_{i j}$ and $K_{i j}$ was used. In the three-node enzyme network design, the error $\mathrm{E}$ achieves the minimum, and then increases monotonically in the neighborhood of the nominal parameters values. The parameters $k_{I A}, k_{F_{A} A}^{\prime}, k_{F_{B} B}^{\prime}$ and $k_{B C}^{\prime}$-representing the input signal, basal enzymes, and the negative regulation in the feedforward loop in Fig. 7(a) - have significant influence on adaptation. Figure 6, however, illustrates that some inconsistent and fluctuating behaviors are present in the four-node enzyme network.

Several factors could account for the phenomenon. In the four-node network, the dynamics are much more complicated than for the three-node system, increasing the possibility of instability or sensitivity. Interactions between variables may also be important. Each trajectory shows only the perturbation of a single parameter while holding other parameters constant. This sensitivity analysis does not quantify variable interactions. 


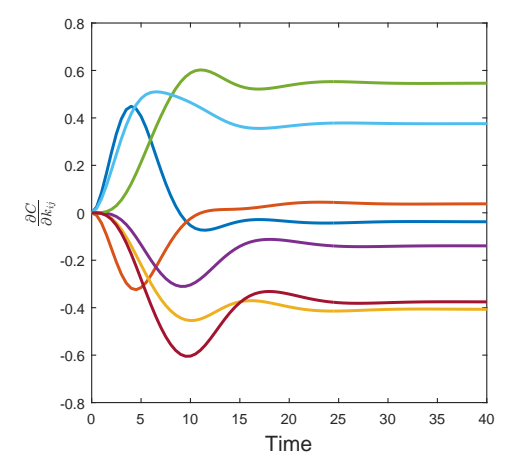

(a) Local sensitivity for $k_{i j}$

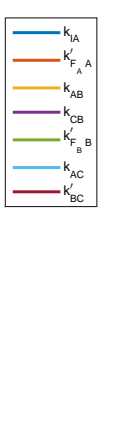



(b) Local sensitivity for $K_{i j}$

Figure 5. Local sensitivity results for the most significant parameters (three-node topology)

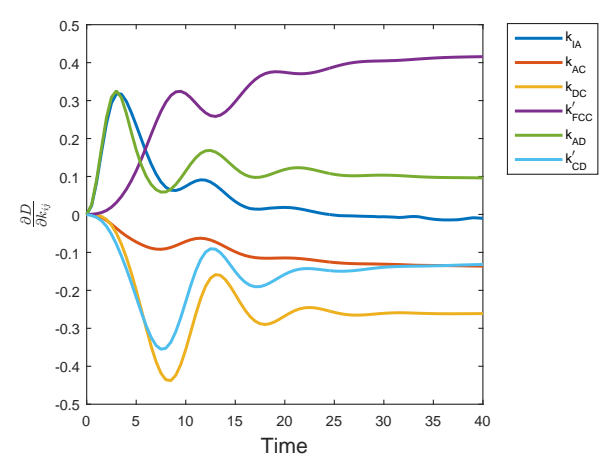

(a) Local sensitivity for $k_{i j}$



(b) Local sensitivity for $K_{i j}$

Figure 6. Local sensitivity results for the most significant parameters (four-node topology)

When performing optimization, however, multiple variables are changed simultaneously. For instance, while the parameter $k_{I A}$ in Fig. 8(a) changes in a undesirable way around the nominal values, the error change still remains within a small range of magnitude $(\leq 0.05)$. It can be seen to be acceptable if one takes into account the complexity of the four-node enzyme network.

A more detailed understanding of system sensitivity to parameter values can be supported by a more detailed parametric sweep that is visualized using gray scale plots. Figures 9-12 show concentration trajectories across a range of perturbation values for the three- and four-node enzyme networks. The green curve indicates the output trajectory (i.e., node $C$ and node $D$ in three- and four-node designs, respectively) corresponding to the optimal design obtained using the simultaneous DT approach. The gray curves illustrate the output trajectories that result from varying the parameter in question at several levels $\pm 20 \%$ from the optimal value.

Several observations can be made regarding the effect of parameter perturbations on network performance. For instance, in Fig. 9(a) (three-node network), decreasing the value of $k_{I A}$ results in larger error because the steady state after the input disturbance at $t=40$ reaches a value that is lower than the steady state value prior to the input disturbance, whereas the optimal green trajectory maintains a reasonable level of error. This can be identified by viewing the black curves in Fig. 9(a). Some other catalytic rate constants parameters such as $k_{F_{A} A}$ and $k_{B C}^{\prime}$ also have significant influence on the output. However, the Michaelis-Menton constants $K_{i j}$ do not exhibit significant influence on the 


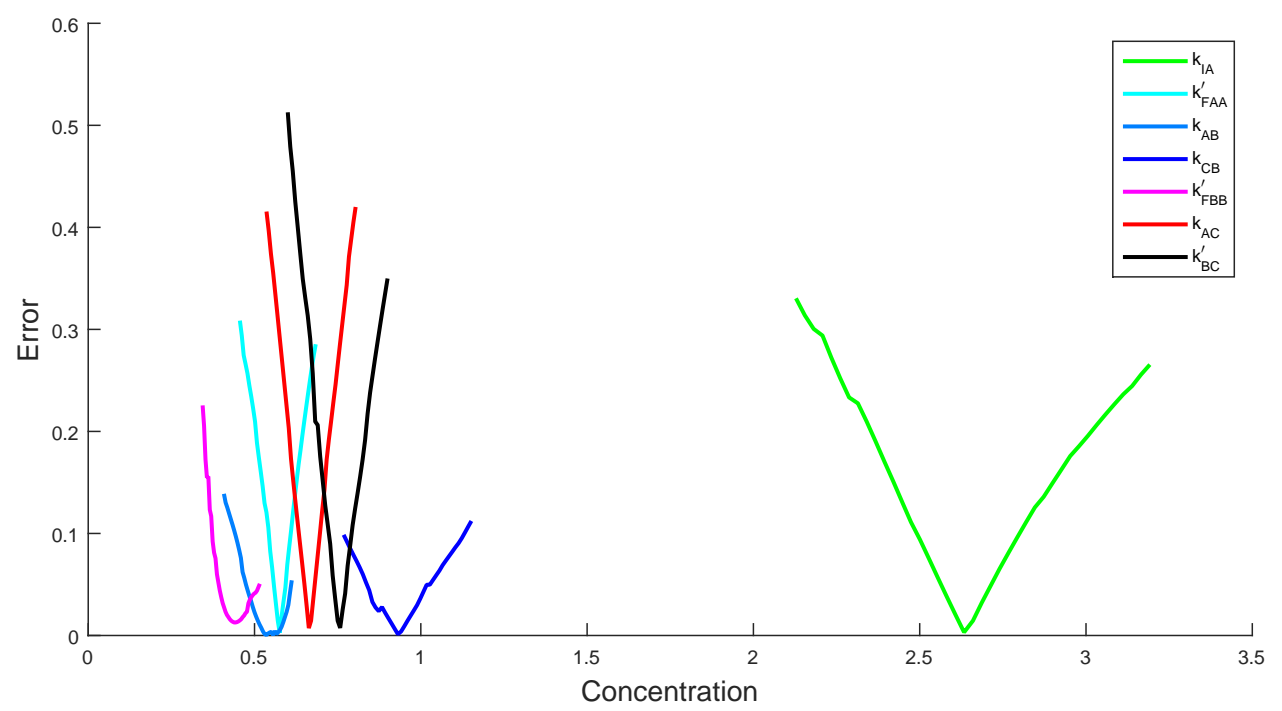

(a) Parameters $k_{i j}$

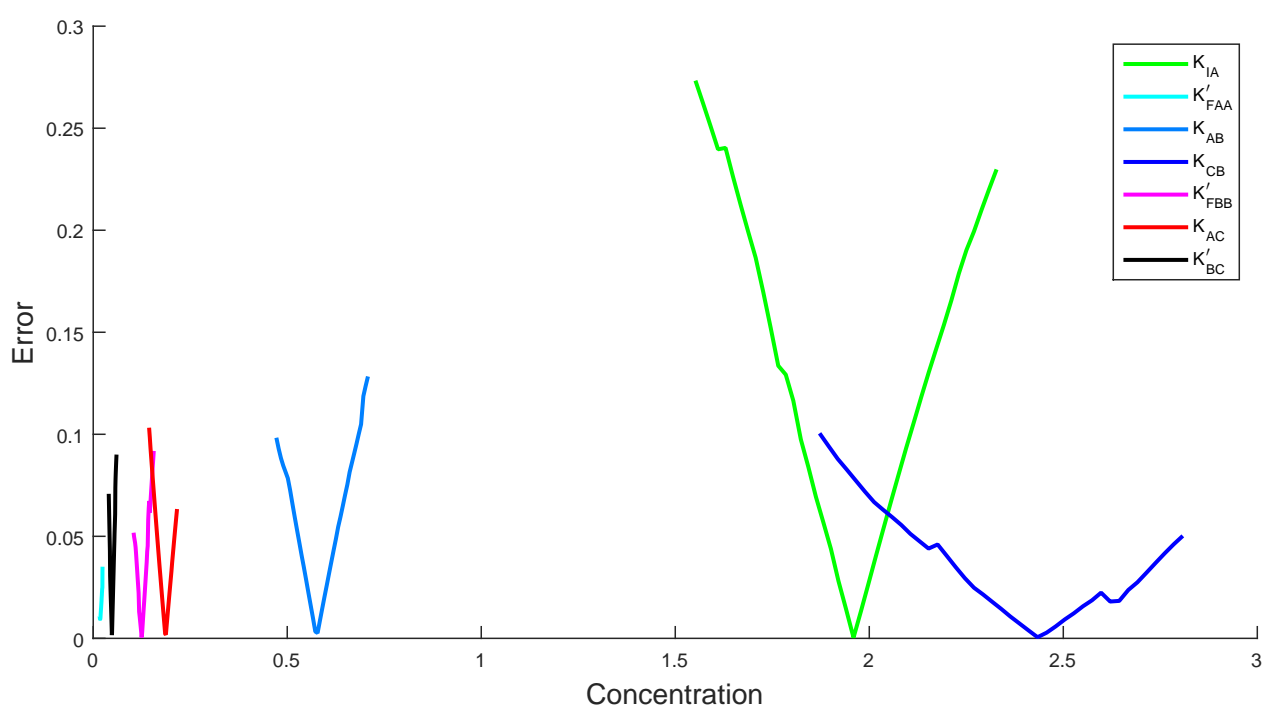

(b) Parameters $K_{i j}$

Figure 7. Error changes with respect to parameter perturbation (three-node topology)

output trajectories for either the three- or four-node networks.

\section{Discussion}

In Ma et al. (2009) all possible three-node topologies (16,038 in total) were enumerated to identify those capable of adaptation. Rather than using optimization to solve the innerloop problem to compare candidate topologies fairly, Ma employed a brute-force approach where 10,000 continuous parameter combinations were tested for each topology. This solution strategy required $\approx 1.6 \times 10^{8}$ distinct enzyme network simulations. While this 


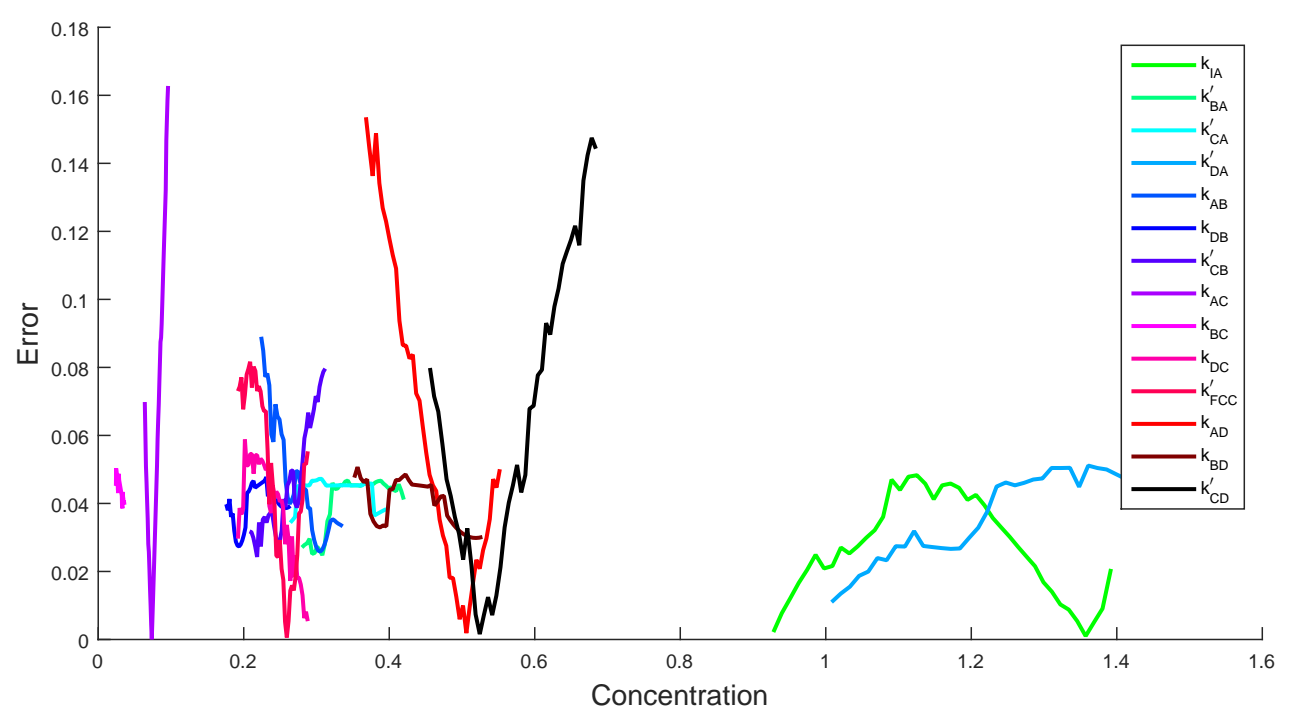

(a) Parameters $k_{i j}$

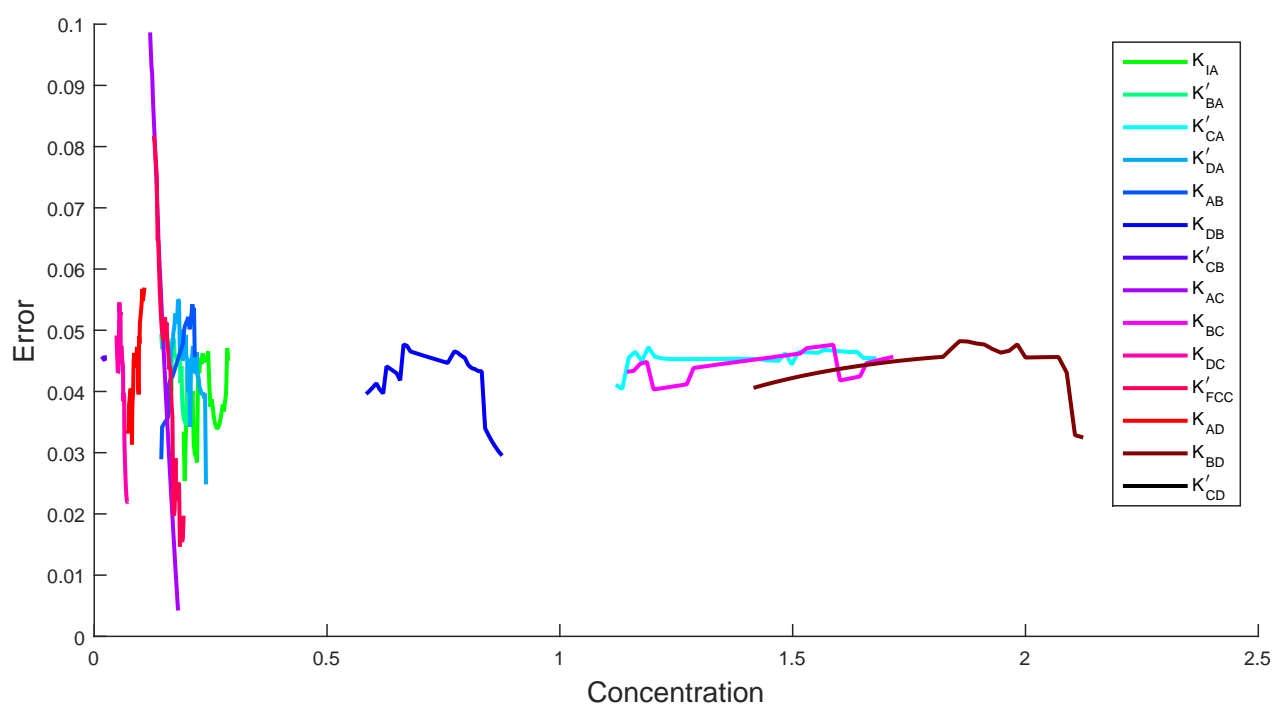

(b) Parameters $K_{i j}$

Figure 8. Error changes with respect to the parameter perturbation (four-node topology)

approach provides comprehensive information about a very low-dimension set of network designs, it does not provide an efficient means to identify particular designs that achieve desired properties. This enumerative strategy is far more computationally expensive than either the nested optimization or simultaneous DT-MPCC solution strategies. In threenode case study, approximately 24,000 and $1.2 \times 10^{5}$ simulations were performed for the DT and single shooting solutions, respectively. While optimization-based strategies are far more efficient for identifying a best design, the enumerative strategy was valuable for providing more general insights regarding the adaptive behavior of three-node networks.

The solutions obtained here confirm the importance of these motifs identified by Ma et al. (2009) in achieving adaptation for three-node networks. For example, negative 
feedback loops and incoherent feed-forward loops were discovered to be important topological features for adaptation in Ma et al. (2009). The path $A \rightarrow B-\bullet C \rightarrow A$ in Fig. 1 (a) is a negative feedback loop based on the single-shooting method. Also, the path $A \rightarrow B \rightarrow C$ and $A \rightarrow C$ in Fig. 2(a) is an incoherent feedforward loop, and associated continuous parameters $k_{i j}$ and $K_{i j}$ will greatly affect the stability of the output due to the high local sensitivity, as discussed in Section 5. These results demonstrate that our DT-MPCC optimization method is capable of identifying important topological motifs in three-node enzyme networks.

In the four-node case, both methods tested successfully produced optimal topologies that achieve adaptation. While DT-MPCC results in a lower scaled precision $\left(P_{*}=0.9568\right)$ compared with the nested optimization method $\left(P_{*}=1.3763\right)$, it is more efficient in identifying the optimal topology, and $P>1$ is not necessary. In nested optimization, the continuous parameters and states must be determined for each candidate topology $\mathbf{x}$ given by the outer loop via inner-loop optimization. The GA generates a sequence of populations of candidate topologies. Parent designs are selected based on design performance determined by the inner loop, and new topology designs are generated based on parent designs using genetic operators such as crossover and mutation (Goldberg 1989). Nested optimization solutions are often more computationally expensive that simultaneous solutions, and the results here are no exception. Simultaneous solution strategies, where possible, often trace a more direct path toward the optimum through the design space. That said, the nested optimization solution is still far more efficient than enumeration, which would require approximately $4.3 \times 10^{7}$ inner-loop solutions. The DT-MPCC method is the most efficient for solving the four-node problem, requiring $\approx 2,500$ simulations. Enumerative solution, even with an optimization-based inner-loop solution, is highly impractical for networks larger than three nodes.

The equilibrium and sensitivity analyses demonstrate that the simultaneous DT approach was able to identify the optimal design topologies and steady states of system responses. While the objective function (error $E$ ) fluctuates with the perturbation of certain parameters in the four-node network case, it does not affect the overall performance of the designed enzyme network. The gray scale plots also imply that the catalytic rate constants $k_{i j}$ have greater influence on the designed network than the Michaelis-Menton constants $K_{i j}$.

\section{References}

Allison, J., 2016. MPCC Solution of Genetic Regulatory Circuit Design Problems. http://www.mathworks.com/matlabcentral/fileexchange/41764-mpcc-solutionof-genetic-regulatory-circuit-design-problems.

Goldberg, D., 1989. Genetic Algorithms in Search, Optimization and Machine Learning. Boston, MA: Addison-Wesley Professional.

Guo, T. and Allison, J.T., 2016. On the Use of Matehmatical Programs wit hComplementarhy Constraints in Combined Topological and Parametric Design of Biochemical Enzyme Networks. Engineering Optimization This reference (main text) needs to be edited by the copyeditor.

Ma, W., et al., 2009. Defining Network Topologies that Can Achieve Biochemical Adaptation.. Cell, 138 (4), 760-773. 


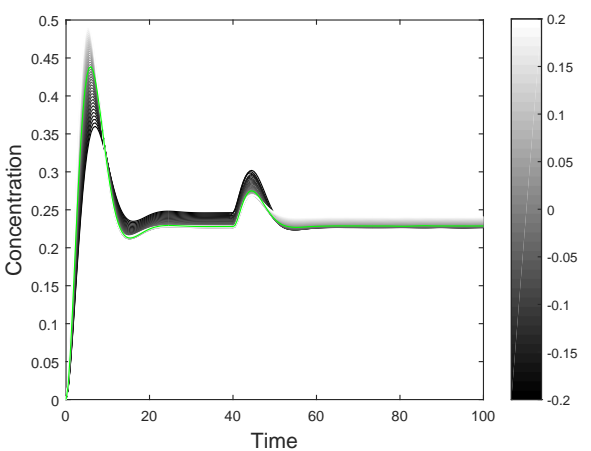

(a) $k_{I A}$



(c) $k_{A B}$

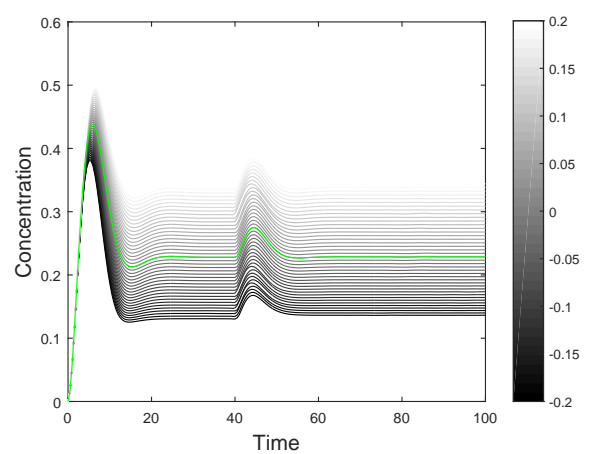

(e) $k_{F_{B} B}^{\prime}$

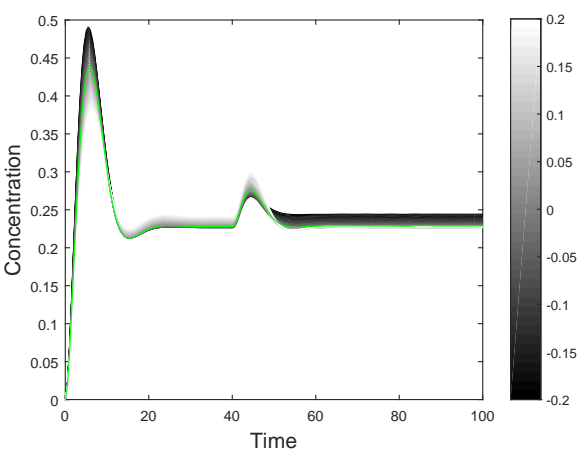

(b) $k_{F_{A} A}^{\prime}$

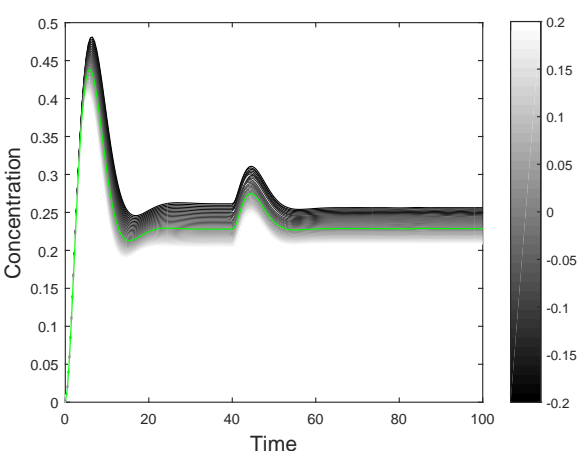

(d) $k_{C B}$

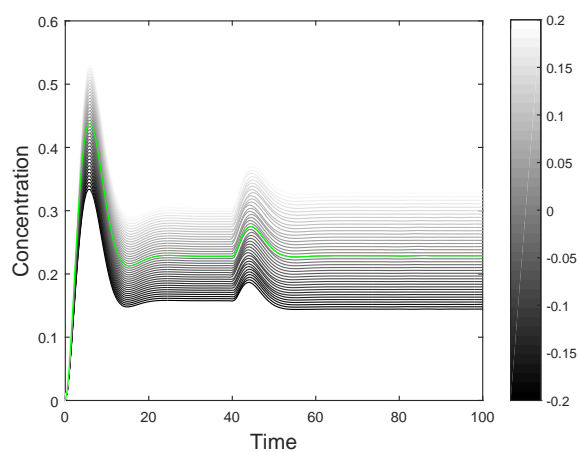

(f) $k_{A C}$

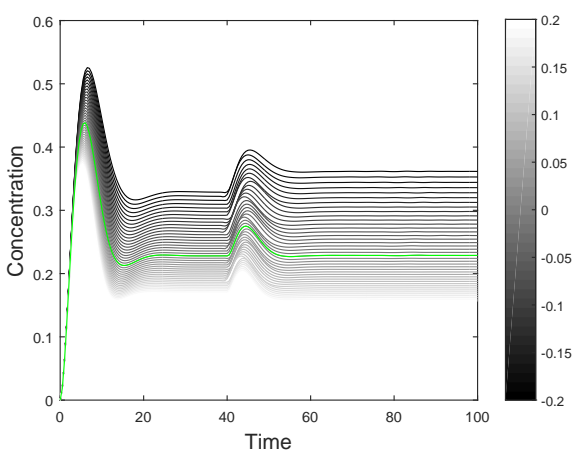

(g) $k_{B C}^{\prime}$

Figure 9. Gray scale plots for perturbed parameter values $k_{i j}$ (3-node topology) 




(a) $K_{I A}$

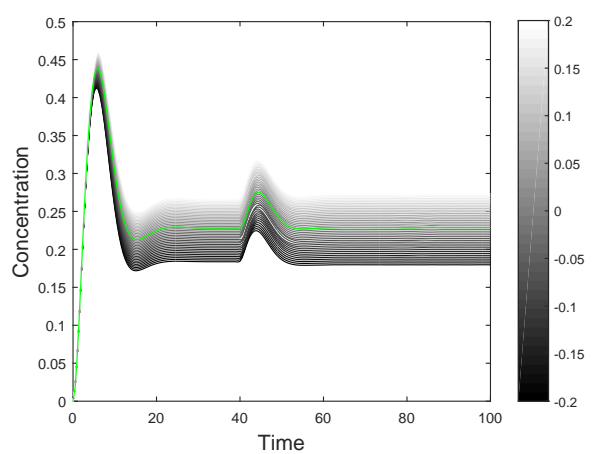

(c) $K_{A B}$

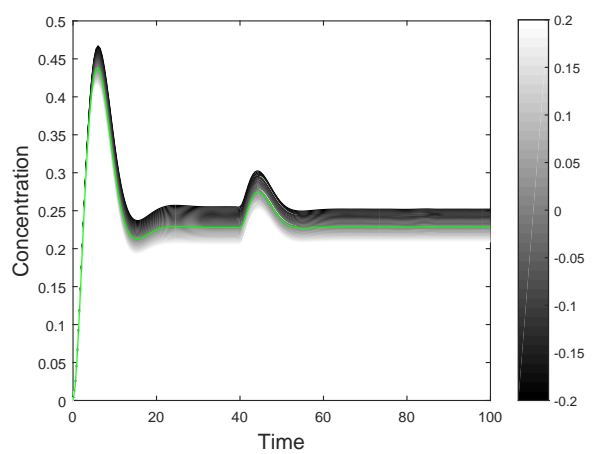

(e) $K_{F_{B} B}^{\prime}$

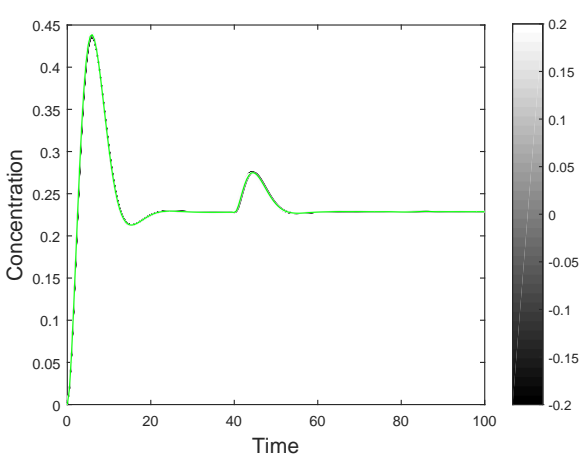

(b) $K_{F_{A} A}^{\prime}$

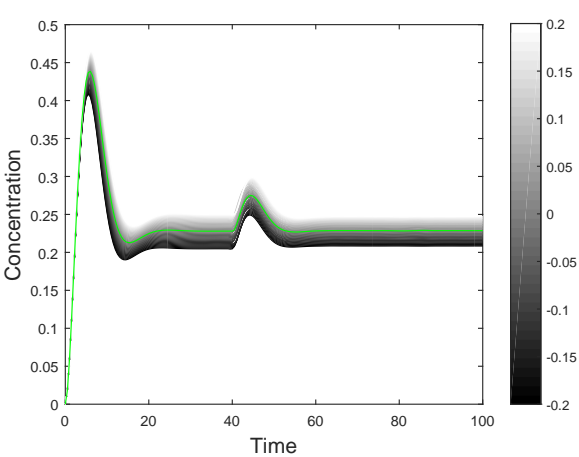

(d) $K_{C B}$

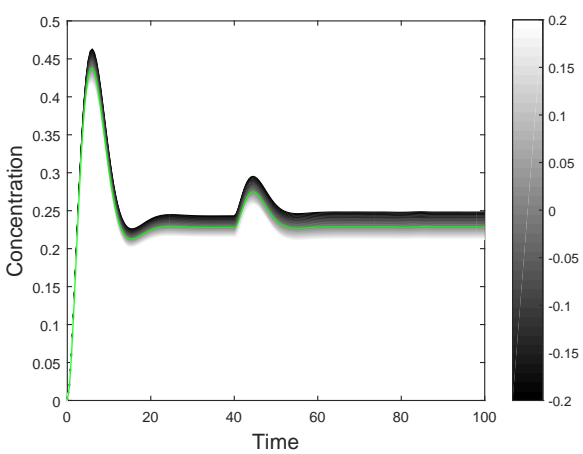

(f) $K_{A C}$



(g) $K_{B C}^{\prime}$

Figure 10. Gray scale plots for perturbed parameter values $K_{i j}$ (3-node topology) 


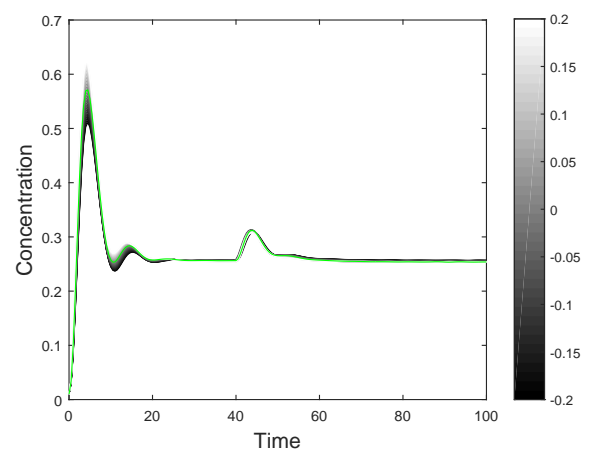

(a) $k_{I A}$

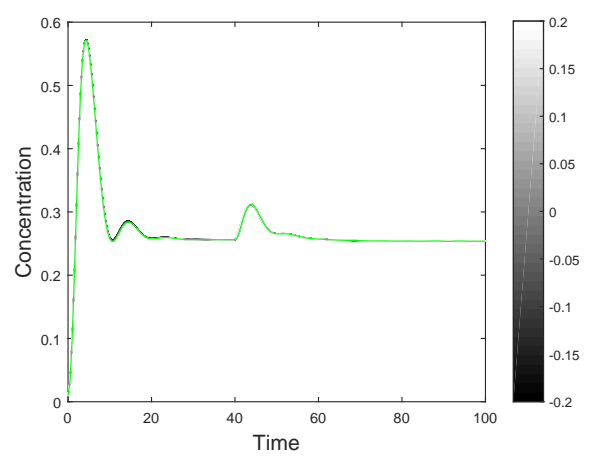

(c) $k_{C A}^{\prime}$

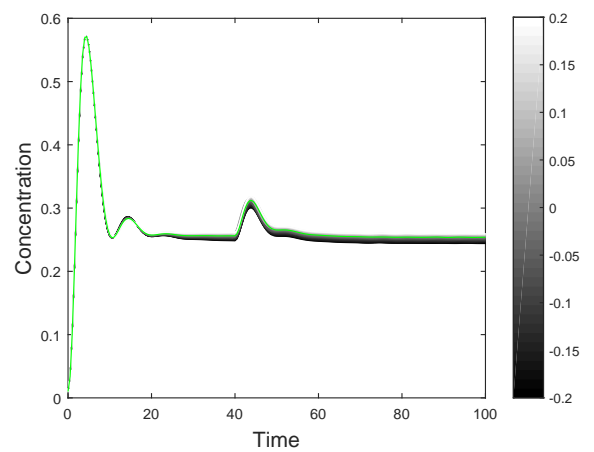

(e) $k_{A B}$

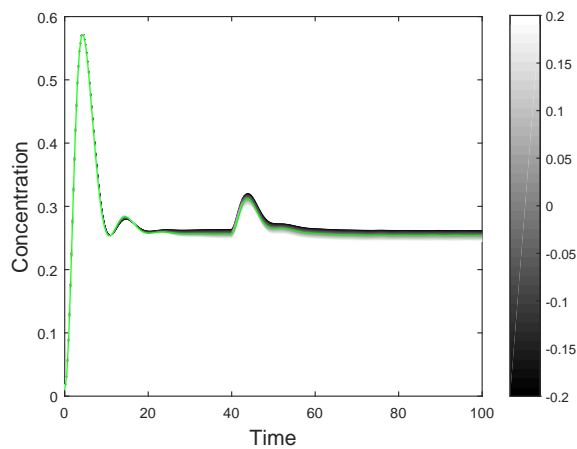

(g) $k_{C B}^{\prime}$

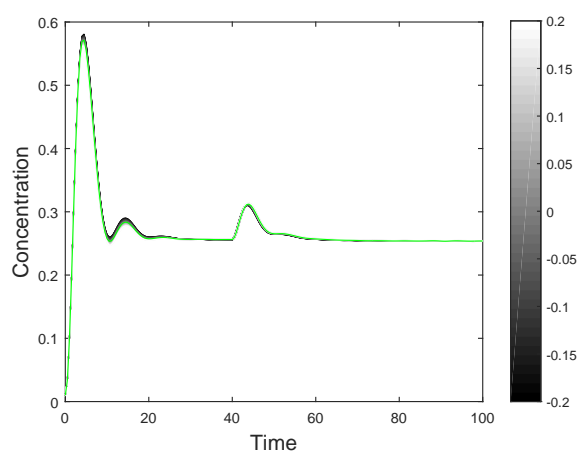

(b) $k_{B A}^{\prime}$

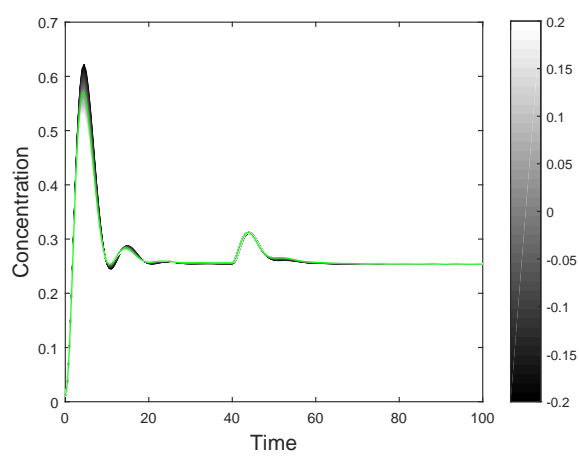

(d) $k_{D A}^{\prime}$

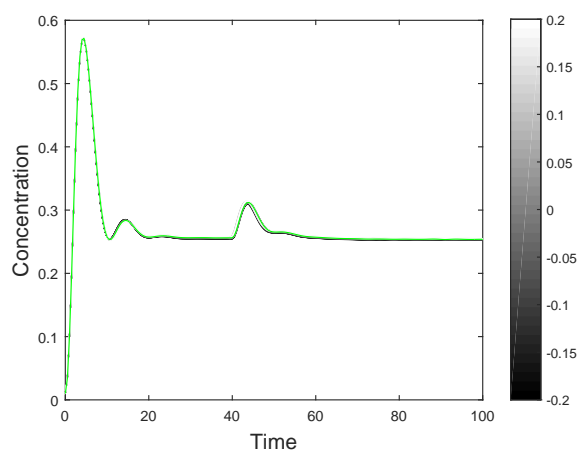

(f) $k_{D B}$

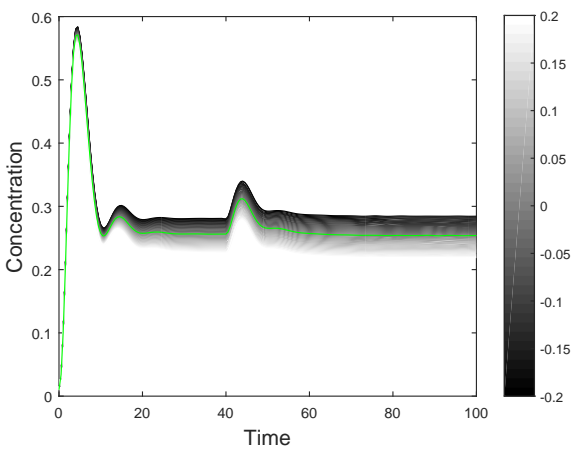

(h) $k_{A C}$ 


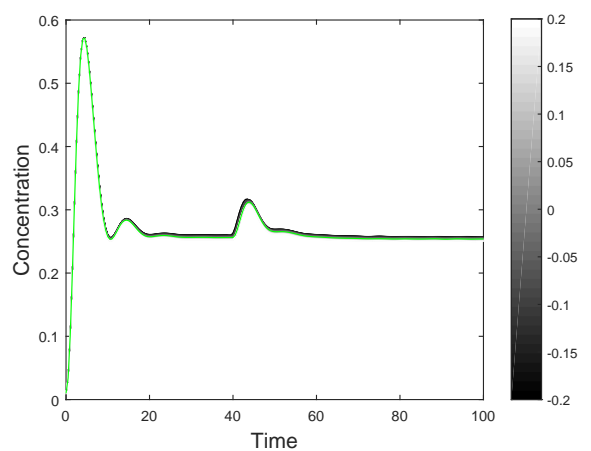

(i) $k_{B C}$

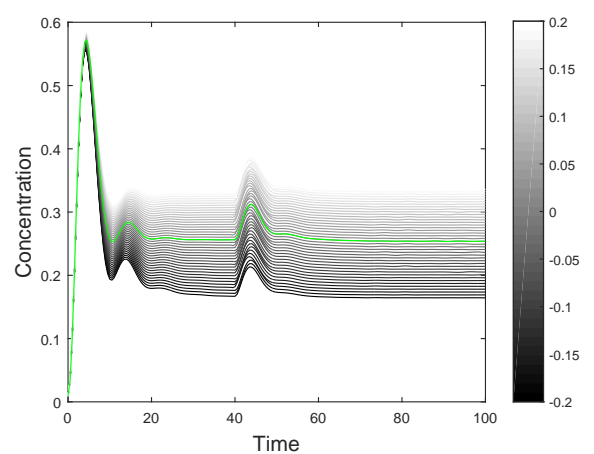

(k) $k_{F_{C} C}^{\prime}$

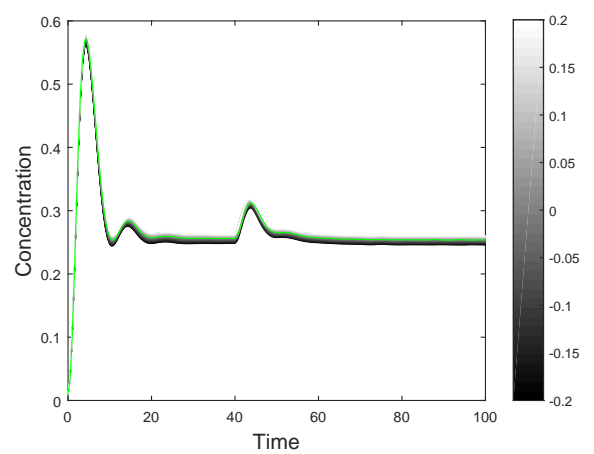

(m) $k_{B D}$

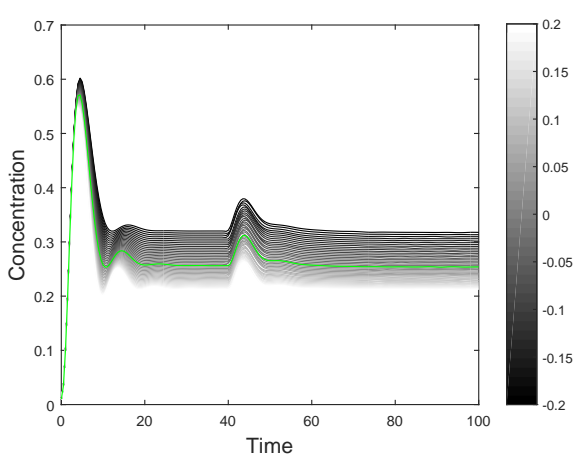

(j) $k_{D C}$

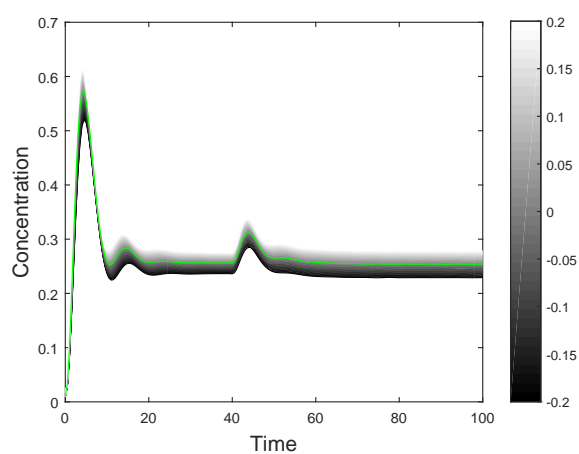

(l) $k_{A D}$

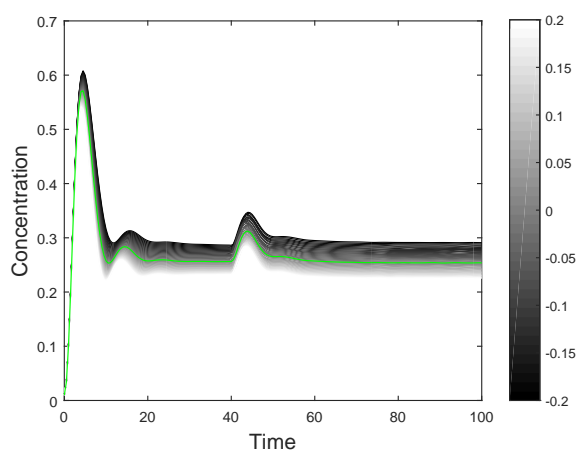

(n) $k_{C D}^{\prime}$

Figure 11. Gray scale plots for perturbed parameter values $k_{i j}$ (4-node topology) 


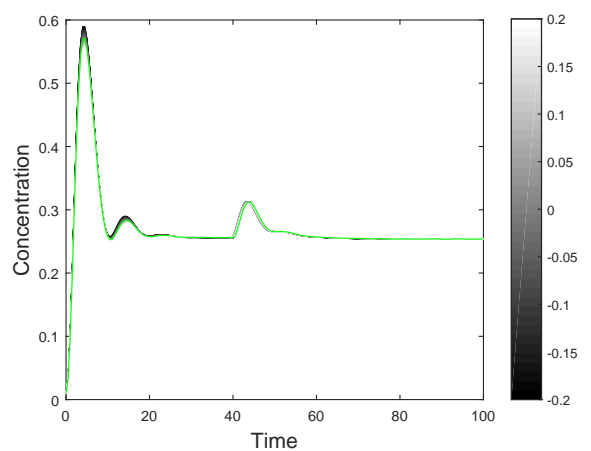

(a) $K_{I A}$



(c) $K_{C A}^{\prime}$

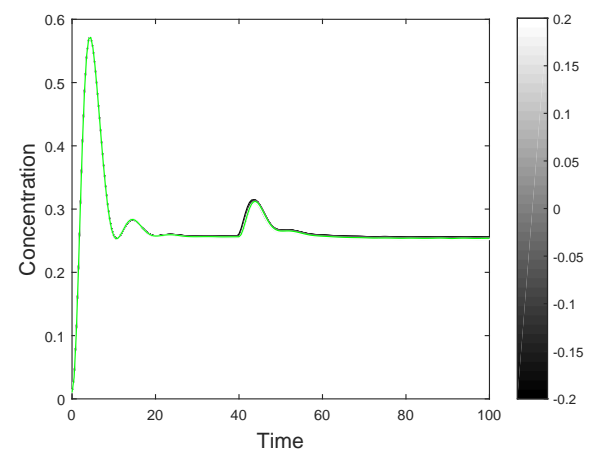

(e) $K_{A B}$

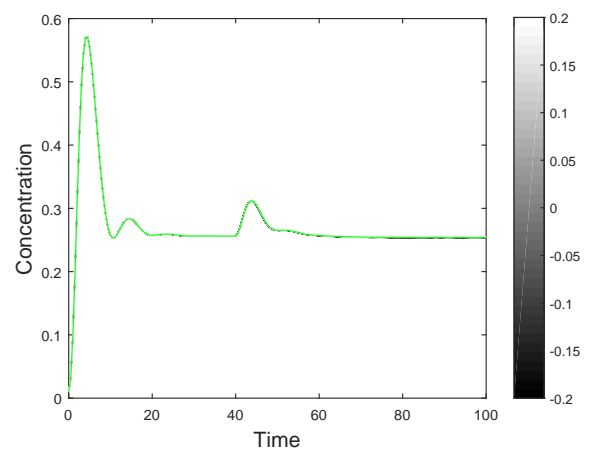

(g) $K_{C B}^{\prime}$

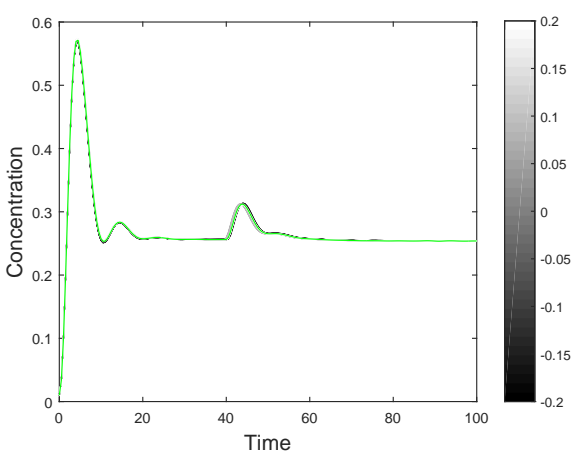

(b) $K_{B A}^{\prime}$



(d) $K_{D A}^{\prime}$

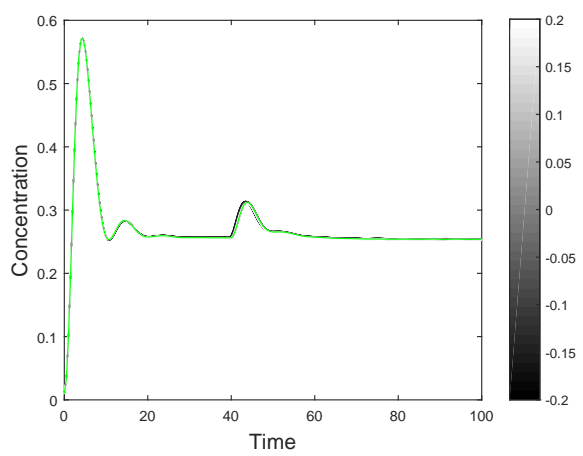

(f) $K_{D B}$

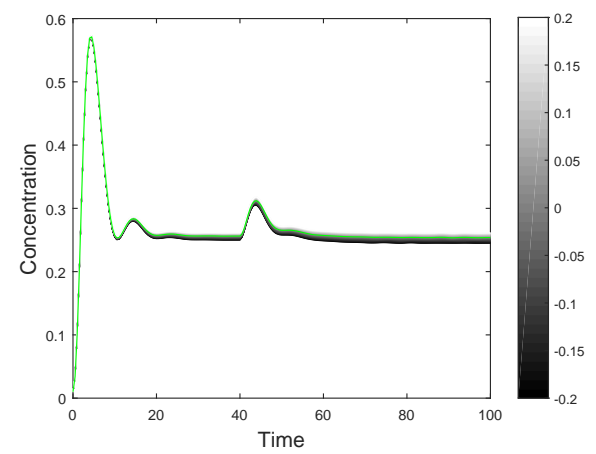

(h) $K_{A C}$ 




(i) $K_{B C}$

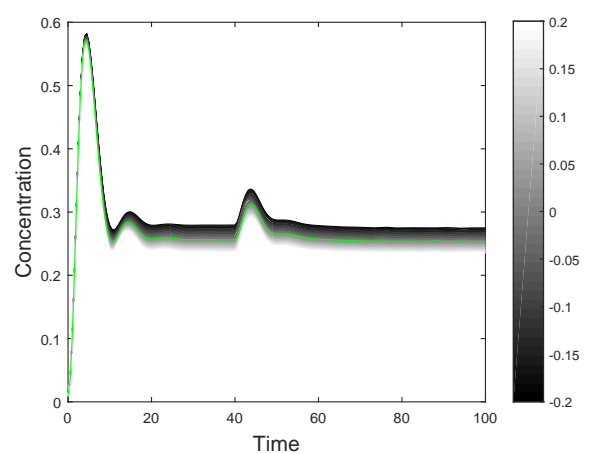

(k) $K_{F_{C} C}^{\prime}$



(m) $K_{B D}$

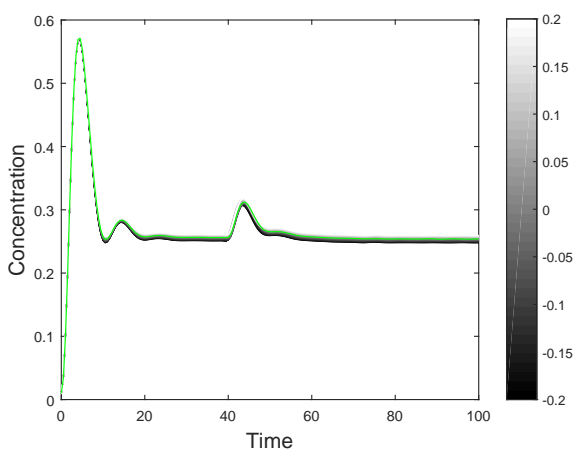

(j) $K_{D C}$

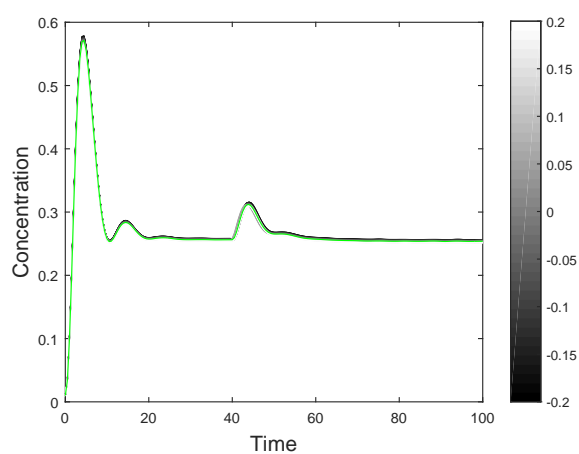

(l) $K_{A D}$

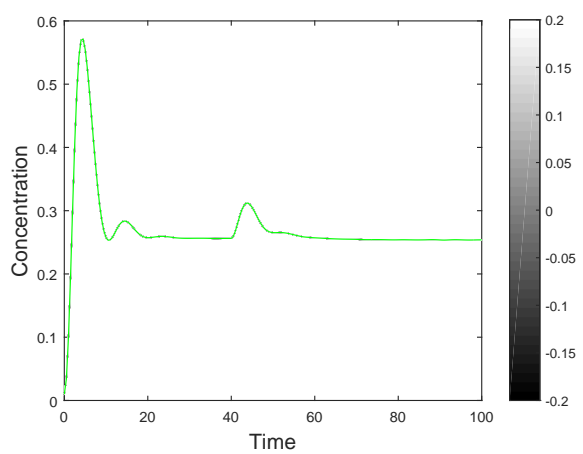

(n) $K_{C D}^{\prime}$

Figure 12. Gray scale plots for perturbed parameter values $K_{i j}$ (4-node topology) 